\title{
The ethical problem of randomization
}

\author{
Agostino Colli $\cdot$ Luigi Pagliaro $\cdot$ Piergiorgio Duca
}

Received: 27 June 2014/ Accepted: 12 August 2014/Published online: 7 September 2014

(C) SIMI 2014

\begin{abstract}
The Fondazione Umberto Veronesi ethics committee recently published a statement concerning the inherent ethical issues of randomized clinical trials (RCTs), mainly focusing on randomization, raising many questions, and suggesting possible solutions. The main concern is that the patients enrolled in a RCT are used to improve medical knowledge, but they cannot be the beneficiaries of the results of the trials in which they are participating. Possible solutions come from a wider use of clinical and administrative databases, and an early termination of trials. We discuss this statement, emphasizing that the scientific and ethical reason for embarking on a clinical trial is uncertainty. The uncertainty regarding the comparative benefits and harms of each compared treatment (clinical equipoise) warrants equity in allocation. Randomization allows one to obtain unbiased evidence that we cannot know in advance. The expected probability of a new treatment to be successful describes the limits within which a study can be acceptable both from an ethical as well as a scientific point of view. Most people accept enrollment in a RCT if the probability of success of the experimental treatment is between 50 and $70 \%$. The assumption and concern that there is a conflict between "scientific" and "ethical"
\end{abstract}

A. Colli $(\bowtie)$

Medical Department, Ospedale A Manzoni AO Provincia di Lecco, Via Eremo 9/11, 23900 Lecco, Italy

e-mail: a.colli@ospedale.lecco.it

L. Pagliaro

Emerito di Medicina, Università degli Studi di Palermo, Palermo, Italy

P. Duca

Department of Biomedical and Clinical Sciences, "L. Sacco," Università degli Studi di Milano, Milan, Italy aspects of a clinical trial due to randomization should at least be mitigated, considering that only scientifically sounded studies can be considered ethical. Randomization remains the appropriate approach to ensure the study's internal validity. Different aspects seem to be more important, from the ethical point of view, considering RCT and their publication.

Randomization is not a scientific method; it is an invaluable statistical strategy for the mathematical exploitation of uncertainty

A.R. Feinstein Ann Intern Med 1994;120:799-805.

\section{Introduction}

The recent statement of the Fondazione Umberto Veronesi ethics committee concerning the role of randomization in clinical research trials is one of the many voices in a wider debate on the necessity of changes in the domain of randomized clinical trials [1]. Since 1948, when the first randomized trial was conducted by Austin Bradford Hill et al., testing the effectiveness of Streptomycin in pulmonary tuberculosis against standard treatment, [2] randomized clinical trials (RCTs) are considered to be the most reliable method for comparing efficacy of different healthcare interventions. However, there are major concerns on the over abundant obstacles in the conduct of these studies, particularly the ever increasing onerous regulation and related bureaucracy that makes them more and more difficult to carry out $[3,4]$. Moreover, from a purely clinical point of view, the major problem is translating the RCT evidence produced "on averages" into clinical decisions regarding individual patients [5-7]. 
The paper from Fondazione Umberto Veronesi focuses upon the inherent ethical issues, mainly randomization, raising many questions, but also proposing possible solutions.

The first question is really basic, it has to do with the ethicality of using patients as tools in reaching a scientific goal. Patients enrolled in a RCT are used to improve medical knowledge, which maybe useful for other patients, but they cannot be the users of the results of the trials in which they are participating. The authors emphasize the fact that the patients assigned to the control arm certainly cannot have any benefit from the new treatment, even if it were to be proven effective. They actually are "discriminated against," given that the study design prevents the patients in the control arm from receiving potentially innovative treatment.

The second question is about the possible harm from the effects of incomplete communication in giving patients information on the treatment actually received, due to the blinding usually associated with randomization. Their consent to the treatment they receive, given in blindness, has an intrinsically lower value.

The third concern deals with the long time needed to complete classically designed RCT, with the consequent delay in making available for clinical practice any treatment that might possibly be more effective than the standard care.

Even if the authors claim to acknowledge the scientific validity, as well as the necessity for randomization, they actually more emphatically emphasize the ethical conflicts, and the consequent need for penetrating solutions, opening the need for a wide discussion. Their suggestions are:

- A wider use of clinical and administrative databases. Databases should be able to store more and more data, faster and faster, which is easily manageable. On this basis, they suggest that clinical (observational) studies should be quicker and simpler, allowing the planning of more focused and ethically correct studies.

- An early (as soon as possible,) termination of every RCT. A RCT should always be planned to be concluded with a minimum number of patients, with a minimum lapse of time, and also consenting with fast availability, for the patients in the control arm of the experimental treatment, if it proves to be effective.

The authors conclude that methodological research for innovative approaches must be encouraged to be increased, and the use of randomization should be reduced. Even if in the discussion there are some attempts to acknowledge different opinions, and some contentious assertions are mitigated, the whole paper seems biased by an excess of optimism, and reliance on the efficacy of new treatments.
We will discuss this statement, and in particular, challenge the points concerning the ethical problem of randomization.

\section{The equipoise principle: genuine uncertainty within the expert medical community about the preferred treatment}

Considering scientific literature, only a minority (less than $15 \%$ ) of experimental treatments evaluated in a clinical context prove to be more effective than standard care [8]. On the contrary, the list of promising and subsequently proved to be detrimental drugs is very long, and the coxib story is only a recent example [9]. Before being introduced into the clinical practice, a new treatment deserves very careful scrutiny, and a very hard and long journey from bench to bedside has to be taken, because it is essential for patient safety. No drug, no intervention, or any diagnostic test as well should be introduced into practice before showing actual effectiveness on relevant clinical outcomes [10]. This requires adequate studies, large samples, and well-conducted RCTs. The evidence of effectiveness of an innovative treatment must never be considered self- proven: it's not enough to be new to be effective. There are no good scientific reasons for claiming a "promising" treatment to be more effective than standard, without appropriate evidence from clinical studies. Moreover, it is even harder to understand why anyone would embark on a RCT if the efficacy of the new treatment was already proven and "glaringly obvious." Any "demonstrative" study has to be considered unethical. Hence, the enrollment into a RCT control arm would be detrimental for patients only if the new treatment had already been demonstrated to be more effective than the comparator. Moreover, such a study would be unethical not because of randomization, but because of futility. The scientific and ethical reasons for embarking on a clinical trial are the actual absence of evidence (clinical equipoise). Randomization, allocation concealment and blinding are the main parameters used in evaluating the quality of a RCT (internal validity), which allow clinicians to obtain sound, unbiased evidence that we cannot know in advance.

From the patient's point of view, this concept cannot be easy to understand, and this misunderstanding could affect patient-physician relationships. Uncertainty is intrinsic to the practice of medicine, even though patients receive a lot of reassuring messages about the accuracy of medical diagnoses and the efficacy of treatments. Learning to cope with uncertainty is critical for clinical reasoning, such as empathically discussing uncertainty with patients for preventing any impairment of their confidence and trust. Administering a treatment according to chance, i.e., 
according to a randomization table, could actually sound very far from either the patient's or the physician's expectations, and even seem to be illogical and unethical, but in the face of actual uncertainty, may lead to the improvement of medical knowledge minimizing possible harm for patients.

On the other side of RCT detractors, there are some enthusiastic supporters who claim that all interventions need to be validated by a randomized controlled trial. In certain cases, the most often quoted being the parachute example, [11], a RCT designed to assess efficacy of an intervention may actually be absurd, and consequently unethical. As was suggested in the putative parachute randomized trial, "Individuals who insist that all interventions need to be validated by a randomized controlled trial need to come down to earth with a bump" [11]. Nevertheless, interventions as effective as is a parachute in preventing "death and trauma related to gravitational challenge," are not abundant, and probably fewer than the authors of the U. Veronesi foundation ethics al committee statement assume [8].

Uncertainty, as a basis for both ethical and scientific reasons in RCTs, is a concept clearly discussed in a recent study in the oncology field. In this careful analysis of the results of RCTs conducted by the National Cancer Institute cooperative groups since their inception in 1955, the pooled estimate of hazard ratios for overall and event-free survival was 0.95 (99 \% CI 0.93-0.98) and 0.90 (99 \% CI, 0.87-0.93) with only a slight favoring of new treatments [12]. These results are consistent with the hypothesis that the ethical principle of equipoise defines limits of discoverability in clinical research, and ultimately drives therapeutic advances in clinical medicine. Planning and conducting these studies, the physicians (as well as the patients), were uncertain about the actual effects of the new versus the standard treatment with which it was to be compared. The expected probability of the new treatment to be successful describes the limits within which a study can be acceptable both from the ethical and the scientific point of view. Most people are willing to be enrolled in a RCT if the probability of success of the experimental treatment is between 50 and $70 \%$, [13] and also most institutional board members would only approve trials with an expected probability of success between 40 and $60 \%$ [14]. These levels of expected probability of success reflect a genuine uncertainty (the equipoise principle), and minimize possible harm for patients in both arms.

\section{The possible harms of randomization}

When the equipoise principle is respected, randomization allows to one to obtain sound unbiased comparisons between treatments. The harms and benefits of being enrolled in a RCT should be balanced, and it is both scientific and ethical to minimize placebo use as opposed to the best standard care of the control arm. A recent systematic review including more than 140,000 patients suggests that participation in RCTs is associated with similar outcomes to receiving the same treatment outside RCTs [15]. Furthermore, some studies even support the hypothesis that hospitals that participate in RCTs compared to hospitals that do not may offer better outcomes [16]. Therefore, it can be argued that RCTs might not be so dangerous, and no specific protection from randomization is needed, whenever the uncertainty principle is respected.

\section{Big data and clinical research}

A wider use of clinical databases, even if very useful in phase four post-marketing studies, cannot replace RCTs: non- randomized comparisons cannot warrant that different outcomes depend on a unique variable i.e., different treatments. In fact, high-quality registries, which collect standardized data from patients seen in a variety of settings, are nowadays available for assessing comparative effectiveness; however, despite statistical advances, observational registry studies are still considered suspect just because they lack the rigor of randomization $[17,18]$. The "by indication bias" is really difficult to eliminate without randomization, even when using the "propensityscore," a statistical approach that allows one to adjust comparisons, minimizing the effect of known possible confounders.

Registry-based randomized trials can offer a solution: investigators are quickly able to identify potential participants by gathering clinical information from preexisting databases, and can enroll thousands of patients in little time, avoiding long case-report forms and obtaining accurate follow-up with minimal effort [19]. An example of such a registry-based trial is the recent Thrombus Aspiration in ST-elevation myocardial infarction in Scandinavia (TASTE) trial, a large-scale study to answer an important clinical question, and carried out at remarkably low cost by building on the platform of an already-existing high-quality observational registry [20]. The trial is still a trial, a rigorous randomized experiment that isolates a causal link (or the absence of one), between a treatment and an outcome. The investigators were able to enroll a large number of patients, thus offering the clinicians' insights that are potentially based on a representative sample, a real-world population created from consecutively enrolled registry patients. This highly efficient design raises some questions that are at the same time ethical and scientific: what are the best populations or sub-populations 
to study to assure representativeness and balance between efficacy versus effectiveness, how to approach concerns about privacy and informed consent, how to assure enough database quality by reducing missing data, and obtaining long-term follow-up? [19]

Furthermore, the problem is not only to hasten studies, but to put them into context: each study should clearly define the pre-existing available evidence to plan an adequate design and build upon a continuous improvement of knowledge, not just a simple sum of data [21]. Hence, one could hypothesize that just the availability of large database and the actual perspective of leading large studies would remove some obstacles and enhance the very use of RCT to solve more and more clinical questions.

\section{The benefit of early stopping RCTs}

Finally, the generalized rule of benefit from the early stopping of RCTs is questionable. A systematic review shows that early stopped trials demonstrate implausibly large treatment effects, particularly when the number of events is small [22]. Because of the overestimation of effect size, and the underestimation of the adverse events rate, an early stopped study cannot support a balanced decision, and ends up being useless, consequently unethical. The definition and planning of stopping rules must prevent patient harm, and also preserve the validity of the results. Turning back to the process of approval of investigational drugs, clarifying the benefit-risk relationship is actually a complex pathway: evidence for both efficacy and safety is needed. Too many attempts to make this pathway shorter produced more harm than benefit. According to the USA experience, fast track or accelerated pathways seem to have only reduced the quantity of needed evidence, and altered the nature of the needed evidence [23]. For example, cancer drugs approved during the previous decade on the basis of limited clinical trials-nonrandomized, unblinded, single group, phase 1 and phase 2 trials that used intermediate end points rather than patient survival-, had a $72 \%$ greater odds of serious adverse events occurring in their pivotal trials than did cancer drugs that were approved with more rigorous studies [24]. Such expedited approvals, with deferral of more rigorous studies, undermine and delay evaluation of the benefit risk profile of an investigational drug. Once a drug is approved, enrolling patients in clinical trials to determine efficacy is more challenging than before approval, because patients have the choice of receiving the drug in the normal course of therapy versus enrolling in a trial in which they may be randomly assigned to usual care [23]. Moreover, it seems difficult for patients to make informed and balanced choice about "breakthrough drugs" approved with new clinical trial techniques rather than with traditional randomized trials, especially considering that it is the very patient with life-threatening illness who seems to underestimate the risk associated with therapy [25].

\section{Research priorities}

Biostatistics research might actually improve studies design in answering clinical questions, but discarding randomization does not seem to be the correct priority. Reducing inconclusive trials, which actually are estimated to be $30 \%$ of all studies [12], might be even more relevant. Such a high level of inefficiency seems to be related to the researchers' overly optimistic assessment of the benefit of treatment rather than to difficulties in the accrual of patients. More realistic estimates of the RCT sample size are needed entailing a clearer definition of the alternative hypothesis (what we mean by: "relevant effect size" and why we want to protect against the "type 1 error" more than against the "type 2 error")? The sample size debate should also take into account the frequent use of metaanalyses [26, 27]. Finally, considering applying summary results of RCT to individual patients, not usually well represented within the "study population" (exclusion by comorbidity), is really of paramount relevance, for methodological, clinical and ethical points of view, to personalize therapy, including in the data analysis, personal risk modeling with appropriate covariates. The aim is not only to adjust comparisons but also to decide on individual patients [7]. Just according to the ethical need of considering patients as the aim of research, a clear definition of patient-centered outcomes should be developed: that is clinical research studies should face problems and questions from the perspective of the patients and their values [28]. Formal statistical significance by itself does not mean clinical relevance, which depends only on patients' actual benefits. Large trials can show differences between the two arms that are statistically significant but clinically not relevant [29]. Even well-conducted RCT do not always properly take into account patients' needs and perspectives: clinical outcomes are not chosen by patients, and may be relevant only for clinicians [30, 31]. Even clinical outcomes such as death or hospitalization are in some sense "intermediate" outcomes, until weighted by patient's preferences.

From the ethical point of view, it seems particularly relevant to pay attention to the sponsorship of the studies, conflict of interest disclosure, authorship and accountability of reporting, completeness of reporting results, even in case of "negative" studies [32, 33]. Sponsorship of drug and device studies by the manufacturing company leads to more favorable results and conclusions than sponsorship by 
other sources [34]. Even the conclusions in trials funded by for-profit organizations may be more positive due to biased interpretation of trial results [35].

\section{Conclusions}

In conclusion, the assumption and concern in the statement of the Fondazione Umberto Veronesi ethics committee that there is a conflict between "scientific" and "ethical" aspects of a clinical trial due to randomization should at least be mitigated, considering that only scientifically sound studies can be considered ethical. Randomization remains a scientific and ethic approach to ensure the internal validity of a study, assuming that the equipoise principle is respected. Other aspects than randomization seem to be more important, from the ethical point of view, considering RCT and their publication [3].

Conflict of interest None.

\section{References}

1. Comitato etico Orientamenti sull'utilizzo della randomizzazione nella sperimentazione clinica. Quaderni Fondazione Umberto Veronesi https://fondazioneveronesi.it/files/1313/8997/2853/Par ere_Comitato_Etico_RANDOMIZZAZIONE_def17gennaio.pdf

2. A Medical Research Council investigation, Streptomycin in tuberculosis Trials Committee (1948) Streptomycin treatment of pulmonary tuberculosis. Br Med J 2(4582):769-782

3. Reith C, Landray M, Devereaux PJ, Bosch J, Granger C, Baigent C, Califf RM, Collins R, Yusuf S (2013) Randomized clinical trialsremoving unnecessary obstacles. New Engl J Med 369:1061-1065

4. Mc Mahon AD, Conway DI, MacDonald TM, McIness GT (2009) The unintended consequences of clinical trials regulation. Plos Med 3:e1000131

5. Greenfield S, Kravitz R, Duan N, Kaplan SH (2007) Heterogeneity of treatment effects: implications for guidelines payment, and quality assessment. Am J Med 120(4A):S3-S9

6. Rothwell PM (2005) External validity of randomised controlled trials: "To whom do the results of this trial apply?". Lancet 365:82-93

7. Kent DM, Hayward RA (2007) Stratification trials to individual patients: the need for risk stratification. JAMA 298(10):12091212 (doi:10.1001/jama.298.10.1209)

8. (2011) BIO/BioMed Tracker clinical trial success rates study. http://insidebioia.files.wordpress.com/2011/02/bio-ceo-biomed tracker-bio-study-handoutfinal-2-15-2011.pdf

9. Coxib and traditional NSAID Trialists (CNT) Collaboration (2013) Vascular and upper gastrointestinal effects of nonsteroidal anti-inflammatory drugs: meta-analyses of individual participant data from randomized trial Lancet (http://www.thelancet.com/ journalslancet/article/PIIS0140-6736(13)60900-9/fulltext)

10. Colli A, Fraquelli M, Casazza G, Conte D, Nikolova D, Duca PG, Thorlund K, Gluud C (2014) The architecture of diagnostic research: from bench to bedside. Research guidelines using liver stiffness as an example. Hepatology 60:408-418. doi:10.1002/hep.26948

11. Smith GCS, Pell JP (2003) Parachute use to prevent death and major trauma related to gravitational challenge: systematic review of randomised controlled trials. BMJ 327:1459-1461
12. Djulbegovic B, Kumar A, Soares HP, Hozo I, Bepler G, Clarke Ml, Bennett CL (2008) Treatment success in cancer new cancer treatment successes identified in phase 3 randomized controlled trials conducted by the National Cancer Institute-Sponsored Cooperative Oncology Groups, 1955 to 2006. Arch Intern Med 168(6):632-642

13. Johnson N, Lilford JR, Brazier W (1991) At what level of collective equipoise does a clinical trial become ethical? J Med Ethics 17(1):30-34

14. Djulbegovic B, Bercu B (2002) At what level of collective equipoise does a clinical trial become ethical for the IRB members? Paper presented at: USF Third National Symposium, Bioethical Considerations in Human Subject Research; March 8, 2002; Clearwater, FL

15. Vist GE, Bryant D, Somerville L, Birminghem T, Oxman AD (2008) Outcomes of patients who participate in randomized controlled trials compared to similar patients receiving similar interventions who do not participate. Cochrane Database of systematic Reviews, Issue 3. Art. No.: MR000009. doi:10.1002/ 14651858.MR000009.pub4

16. Majumdar SR, Roe MT, ED Peterson, Chen AY, Gibler WB, Armstrong PW (2008) Better outcomes for patients treated at hospitals that participate in clinical trials. Arch Intern Med 168(6):657-662

17. Pocock SJ, Elbourne DR (2000) Randomized trials or observational tribulations? N Engl J Med 342:1907-1909

18. Drazen JM, Gelijns AC (2014) Statin Strikeout. Engl J Med. doi:10.1056/NEJMe1405032

19. Lauer MS, D'Agostino RB (2013) The randomized registry trial-the next disruptive technology in clinical research? N Engl J Med 369:1579-1581

20. Fröbert O, Lagerqvist B, Olivecrona GK et al (2013) Thrombus aspiration during ST-segment elevation myocardial infarction. N Engl J Med 369:1587-1597

21. Young C, Horton R (2005) Putting clinical trials into context. Lancet 366:107-108

22. Montori VM, Devereaux PJ, Adhikari NKJ, Burns KEA, Eggert $\mathrm{CH}$, Briel M, Lacchetti C, Leung TW, Darling E, Bryant DM, Bucher HC, Schünemann HJ, Meade MO, Cook DJ, Erwin PJ, Sood A, Sood R, Lo B, Thompson CA, Zhou Q, Mills E (2005) Guyatt, GH Randomized trials stopped early for benefit. A systematic review. JAMA 294:2203-2209

23. Darrow JJ, Avorn J, Kesselheim AS (2014) New FDA breakthrough-drug category-implications for patients. New Engl J Med 370:1252-1258

24. Kesselheim AS, Myers JA, Avorn J (2011) Characteristics of clinical trials to support approval of orphan vs nonorphan drugs for cancer. JAMA 305:2320-2326

25. Schaeffer MH, Krantz DS, Wichman A, Masur H, Reed E, Vinicky JK (1996) The impact of disease severity on the informed consent process in clinical research. Am J Med 100:261-268

26. Guyatt GH, Mills EJ, Elbourne D (2008) In the era of systematic reviews, does the size of an individual trial still matter? Plos Med 5(1):e4

27. Wetterslev J, Thorlund K, Brok J, Gluud C (2008) Trial sequential analysis may establish when firm evidence is reached in cumulative meta-analysis. J Clin Epidemiol 61:64

28. Public comment draft report of the patient-Centered Outcomes Research Institute (PCORI) Methodology Committee presented on July 23 (2012). http://www.pcori.org

29. Sobrero A, Bruzzi P (2009) Incremental advance or seismic shift? The need to raise the bar of efficacy for drug approval. J Clin Oncol 27(35):5868-5873

30. Devereaux PJ, Fahey T, Anderson DR, Gardner MJ, Putnam W, Flowerdew GJ, Brownell BF, Nagpal S, Cox JL (2001) 
Differences between perspectives of physicians and patients on anticoagulation in patients with atrial fibrillation: observational study. BMJ 323:1218-1222

31. Rothwell PM, McDowell Z, Wong CK, Dorman PJ (1997) Doctors and patients don't agree: cross sectional study of patients' and doctors' perceptions and assessments of disability in multiple sclerosis. BMJ 314:1580-1583

32. Manzoli L, Flacco ME, D'Addario M, Capasso L, De Vito C, Marzuillo C, Villari P, Ioannidis JP (2014) A Non-publication and delayed publication of randomized trials on vaccines: survey. BMJ 348:g3058. doi:10.1136/bmj.g3058
33. Ladher N (2014) Secrets in healthcare. BMJ 348:g3439

34. Lundh A, Sismondo S, Lexchin J, Busuioc OA, Bero L (2012) Industry sponsorship and research outcome. Cochrane Database of Systematic Reviews. Issue 12, Art. No.: MR000033. doi:10. 1002/14651858.MR000033.pub2

35. Als-Nielsen B, Chen W, Gluud C, Kjaergard LL (2003) Association of funding and conclusions in randomized drug trials. JAMA 290:921-928

36. Smith R (2005) Medical journals are an extension of the marketing arm of pharmaceutical companies. PLoS Med 2:e138 